\title{
Grau de Aderência após Ressecção Parcial e Reconstituição do Ovário
}

\author{
Adhesion after Partial Resection and Ovarian Reconstruction of the Ovary \\ Marco Aurelio Pinho de Oliveira ${ }^{1}$, Manuel Domingos da Cruz Gonçalves ${ }^{2}$ \\ Nelson $\mathrm{Jamel}^{2}$, Célio Pacheco Alves ${ }^{2}$
}

\begin{abstract}
RESUM0
Objetivo: comparar o grau de aderências no ovário de acordo com diferentes tipos de fechamento do córtex ovariano.

Métodos: quinze coelhos foram distribuidos aleatoriamente em três grupos, de acordo com o tipo de fechamento do córtex ovariano, sendo utilizados a diatermia bipolar, a poliglactina 910 5-0 e o categute simples 5-0. Após 2 semanas os animais foram sacrificados e o grau de aderências do ovário direito foi avaliado segundo a classificação de Diamond. O ovário esquerdo, não incisado, serviu de controle. Na análise estatística foram empregados a análise de variância (ANOVA), o teste de Scheffé e o teste t de Student.

Resultados: as médias dos escores de aderência para o grupo bipolar, poliglactina e categute simples foram 0,7, 1,5 e 2,0, respectivamente. A análise de variância detectou diferença significativa $(p=0,02)$ entre os três grupos. Usando-se o teste de Scheffé para a comparação dos grupos dois a dois, encontrou-se uma diferença significativa apenas entre o grupo bipolar e o grupo do categute simples. Comparando-se o grupo em que se usaram suturas (independente do material empregado) com o grupo bipolar verificou-se uma diferença estatisticamente significativa $(p=0,01)$ entre as médias dos escores de aderência do ovário direito $(1,8$ e 0,7 , respectivamente).

Conclusões: os resultados obtidos no presente estudo estão de acordo com os dados da literatura, indicando que o fechamento por segunda intenção do córtex ovariano é mais vantajoso, em relação à formação de aderências, que o fechamento com o uso de suturas.
\end{abstract}

PALAVRAS-CHAVE: Peritônio. Infertilidade. Ovário: aderências. Modelo experimental.

\section{Introdução}

A gônada feminina é um órgão que comumente apresenta doenças que requerem o tratamento cirúrgico, e entre as mais importantes estão os cistos ovarianos. Qualquer cirurgia tem o risco potencial de causar aderências e isto também é verdadeiro para as cirurgias ovarianas, já que a superfície ovariana é a que mais freqüentemente apresenta aderências na pelve ${ }^{1}$. Apesar de uma técnica cirúrgica cuidadosa, as ade-

\footnotetext{
1 Serviço de Ginecologia do Hospital Universitário Pedro Ernesto - Faculdade de Ciências Médicas da Universidade do Estado do Rio de Janeiro

2 Departamento de Cirurgia do Hospital Universitário Clementino Fraga Filho - Universidade Federal do Rio de Janeiro

Correspondência: Marco Aurelio Pinho de Oliveira

R. Coelho Neto 55 / 201 - Laranjeiras

22231-110 - Rio de Janeiro - RJ
}

rências podem ser identificadas, por laparoscopia, em cerca de $50 \%$ das pacientes submetidas à cirurgia anexial por laparotomia ${ }^{2}$. A extensão e a densidade das aderências parecem aumentar com o passar do tempo após a realização do procedimento cirúrgico ${ }^{3}$.

Deve-se também levar em conta que a maioria das mulheres encontra-se no menacme, período que coincide com a idade fértil feminina. As aderências podem causar infertilidade por vários mecanismos, como o bloqueio da tuba uterina, a distorção da anatomia tubo-ovariana, a incapacidade da tuba em captar o óvulo, a fixação do útero em posição anormal e o bloqueio do ovário, interferindo no mecanismo ovulatório ${ }^{4}$.

Vários autores mostraram que a presença de aderências periovarianas dificultam a gravidez e que com a microcirurgia realizada para lise destas aderências se obtêm resultados significa- 
tivamente melhores quando as pacientes apresentam aderências frouxas e em pequena quantida$\mathrm{de}^{5-7}$.

Em mulheres submetidas à fertilização in vitro com transferência de embriões, o efeito das aderências periovarianas não é tão evidente. Alguns autores afirmam que essas aderências interferem na foliculogênese ${ }^{8}$, ao passo que outros não conseguiram demonstrar tal efeito ${ }^{9}$. De um modo geral, as aderências periovarianas são responsáveis por cerca de $20 \%$ dos casos de infertilidade ${ }^{10}$.

Devido ao efeito deletério das aderências periovarianas sobre a fertilidade, o cirurgião deve fazer o possivel para evitá-las. Para isso, deve levar em conta mais a precisão da cirurgia do que o uso de substâncias teoricamente protetoras. O uso de suturas para o fechamento das feridas pode inadvertidamente levar a uma maior freqüência de formação de aderências por causar isquemia focal $^{11}$.

Rotineiramente, os ginecologistas utilizam suturas para o fechamento do córtex ovariano no tratamento do cisto de ovário. O uso de fios pode ser prejudicial, propiciando a formação de mais aderências, com conseqüente prejuízo na fertilidade feminina. Apesar de não estar comprovado que as aderências causem dor, elas estão presentes em cerca de $25 \%$ a $50 \%$ das pacientes com dor pélvica crônica ${ }^{12}$.

O objetivo do presente estudo é comparar o grau de aderência no ovário de acordo com o tipo de fechamento do córtex ovariano. A nossa hipótese é de que o fechamento por segunda intenção causa menos aderências quando comparado ao fechamento com suturas.

\section{Material e Métodos}

Foram selecionadas 15 coelhas do tipo Nova Zelândia (Orytolagus ciniculus) para o experimento. Todas se encontravam saudáveis e pesavam entre $2.500 \mathrm{~g}$ e $3.500 \mathrm{~g}$. Foram divididas em cinco blocos de três coelhas. Cada bloco era adquirido 72 horas antes da primeira cirurgia, sendo que cada animal era colocado aleatoriamente em gaiolas separadas (numeradas de um a três) e recebia, neste momento, 200 UI de gonadotrofina coriônica $(0,2 \mathrm{ml}$ de Profasi $\mathrm{HP} \circledast, 1000 \mathrm{UI})$ intramuscular. Para assegurar a estabilidade do produto foram utilizadas soluções recém-preparadas para cada administração. As gaiolas ficavam numa sala do biotério, observando-se a temperatura em torno de $25^{\circ} \mathrm{C}$ (ar condicionado). Os animais recebiam ração e água à vontade, até o mo- mento da cirurgia. No dia da cirurgia, os animais eram operados na ordem crescente de numeração das gaiolas.

Antes de serem levados para a sala de cirurgia, cada animal recebia uma dose de $50 \mathrm{mg} /$ $\mathrm{kg}$ de quetamina intramuscular. Já na sala, era instalada a hidratação venosa com soro fisiológico, puncionando-se com agulha bem fina a veia localizada na borda lateral de uma das orelhas. Essa via permitia a infusão adicional de quetamina, conforme a necessidade, avaliada pelo anestesista. O plano anestésico era mantido com halotano, fornecido em pequena máscara adaptada para uso em coelhos. Após tricotomia da região abdominal infra-umbilical, o animal era posicionado de forma a permitir o uso do microscópio cirúrgico. A utilização do microscópio (aumento de 6 vezes) permite um manuseio mais delicado, pois o ovário do coelho mede aproximadamente $1,2 \mathrm{~cm}, 0,8 \mathrm{~cm}$ e $0,6 \mathrm{~cm}$ em comprimento, largura e altura, respectivamente.

O cirurgião e o auxiliar vestiam gorro, máscara e calçavam luva, tendo o cuidado de lavá-las antes da cirurgia, para evitar a contaminação peritoneal com talco ou amido. A seguir, era feita anti-sepsia em toda região abdominal infra-umbilical com Povidine tópico e colocava-se um campo fenestrado para deixar exposta somente a área a ser operada. Não foram utilizados antibióticos profiláticos.

Em cada animal foi realizada uma incisão mediana com bisturi cabo no 3 e lâmina no 15 , iniciando-se a $1 \mathrm{~cm}$ da cicatriz umbilical e estendendo-se inferiormente por aproximadamente 4 $\mathrm{cm}$. O tecido subcutâneo, a fáscia, o músculo e o peritônio eram abertos por planos, fazendo-se hemostasia rigorosa. Ao se entrar na cavidade pélvica, o útero (bicorno) era identificado e ia-se em busca do ovário direito, seguindo-se a trompa ipsilateral. Neste momento, o ovário era fixado pelo assistente e o auxiliar de sala posicionava o microscópio para obtenção de melhor campo e foco.

O ovário direito era seccionado com lâmina de bisturi no intuito de se extrair em torno de $30 \%$ do parênquima ovariano. A retirada do material era facilitada com o uso de uma pequena tesoura e de uma pinça dente-de-rato delicada. A técnica de fechamento variava ordenadamente (da mesma forma para cada um dos 5 blocos, com 3 coelhas cada):

No 1a animal, a hemostasia era obtida com coagulação microbipolar (Wavetronics 400), com a potência colocada em cinco $(50 \%$ da potência máxima), o suficiente para obtenção de uma boa hemostasia sem coagular áreas desnecessárias. O parênquima era deixado aberto para fechar por segunda intenção. Não se utilizou sutura neste 
grupo.

No $2^{\circ}$ animal, a hemostasia e o fechamento eram conseguidos com o uso de um fio de poliglactina 910 (Vicryl $\AA$ ) 5-0 com agulha cilindrica de $1,5 \mathrm{~cm}$. A sutura usada foi a contínua nãoentrelaçada, visando apenas a área cortical do ovário.

No 3o animal, a hemostasia e o fechamento eram obtidos com o uso de um fio de categute simples $5-0$ com agulha cilindrica de $1,5 \mathrm{~cm}$. A sutura usada foi a continua não-entrelaçada, visando apenas a área cortical do ovário.

O passo seguinte era idêntico para os três grupos: o ovário esquerdo era manipulado fora da cavidade pélvica por um tempo aproximado ao dispensado no ovário operado. A intenção era submeter ambos os ovários à mesma possibilidade de aderências causada somente pela realização da laparotomia.

O peritônio, a fáscia, o músculo e a pele eram fechados em plano único com poliglactina 3-0, usando-se sutura contínua não-entrelaçada.

Os animais retornavam às gaiolas conforme numeração prévia. Após duas semanas, os animais eram sacrificados com pentobarbital 1 $\mathrm{mg} / \mathrm{kg}$ intravenoso. A avaliação do grau de aderências era feita sem que o autor soubesse qual o número do animal selecionado. A escolha era feita aleatoriamente pelos assistentes (anotavam o número do grupo num papel e mantinham sigilo durante a reoperação).

$\mathrm{Na}$ reoperação, a parede abdominal era aberta por planos, usando-se a mesma incisão da cirurgia inicial. Após entrada na cavidade abdominal, as alças intestinais eram afastadas da pelve com um chumaço de gaze. Iniciava-se a avaliação do grau de aderências ovarianas pela gônada direita (operada previamente), empregando a classificação de Diamond $^{1}$, que foi idealizada para avaliação de aderências ovarianas em coelhos. São atribuidos percentuais de aderências de acordo com a área de ocupação das mesmas em relação à superfície ovariana (porção medial, linha da incisão e porção lateral). O percentual ocupado pelas aderências em cada uma dessas três áreas é somado e o total anotado (pontuação máxima de $3,00)$. Os tipos de aderências (frouxas ou densas) são anotados mas não computados.

Após a classificação das aderências do anexo direito, era feita a classificação das aderências do ovário esquerdo (não-operado, mas manipulado), usando-se o mesmo critério da gônada contralateral. A parede abdominal era fechada em plano único e o corpo do animal era ensacado e colocado em lixo apropriado do biotério.

Após o término da avaliação do escore de aderências dos cinco blocos (total de quinze coelhos), as fichas de classificação das aderências foram abertas e foi feita a análise estatística, empregando-se a análise de variância (ANOVA) para os escores obtidos com a classificação de Diamond (escala intervalar com distribuição normal), considerando-se significativo $\mathrm{p}<0,05$. Após teste global significativo (para os três grupos), foi feita a comparação dois a dois entre os três grupos, usando-se o teste de Scheffé. Para a análise comparativa da média dos escores das aderências do grupo com suturas (não levando em consideração o tipo de fio) com a do grupo bipolar foi utilizado o teste $t$ de Student.

\section{Resultados}

No momento da laparotomia inicial, nenhum dos ovários apresentava qualquer grau de aderência. Na segunda laparotomia, entre os 15 ovários manipulados mas não-operados (lado esquerdo) foram encontrados apenas dois casos com valor acima de zero (ou seja, com aderências) na classificação de Diamond, sendo os escores destes dois animais de 0,3 no grupo da poliglactina e 0,4 no grupo do categute simples.

As Figuras 1, 2 e 3 mostram os diversos graus de aderências encontrados no ovário direito (operado) no momento da segunda laparotomia. A Tabela 1 mostra o escore de aderências de cada ovário direito, de acordo com o tipo de fechamento e bloco de randomização. As médias dos escores para o grupo bipolar, poliglactina e categute simples foram $0,7,1,5$ e 2,0 respectivamente. A análise de variância detectou diferença significativa ( $p=0,02)$ entre os três grupos. Usando-se o teste de Scheffé para a comparação dos grupos dois a dois, encontrou-se uma diferença significativa apenas entre o grupo bipolar e o grupo do categute simples (Tabela 2).

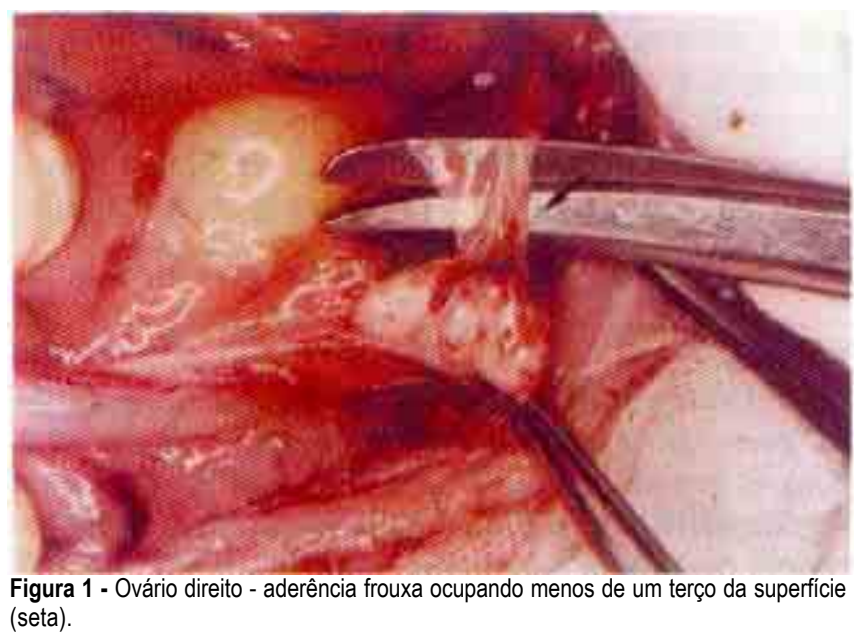




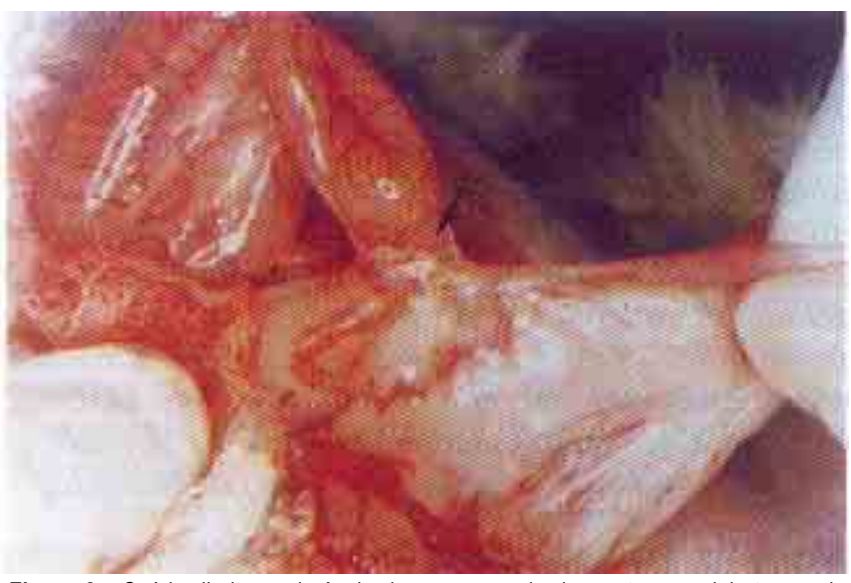

Figura 2 - Ovário direito - aderência densa ocupando de um terço a dois terços da superfície (seta).

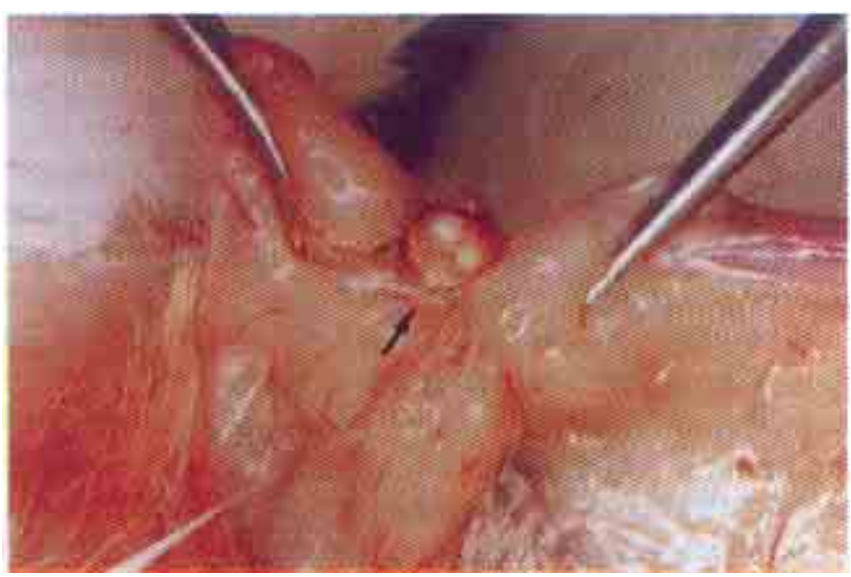

Figura 3 - Ovário direito - aderência densa ocupando mais de dois terços da superfície (seta).

Tabela 1 - Escores de aderências de cada ovário direito, de acordo com o bloco de randomização e o tipo de fechamento do córtex.

\begin{tabular}{lccc}
\hline Bloco & \multicolumn{3}{c}{ Tipo de Fechamento } \\
& Bipolar & Poliglactina & Categute simples \\
\hline 1 & 0,4 & 2 & 2,8 \\
2 & 0,9 & 1,5 & 1,1 \\
3 & 0,6 & 0,6 & 2,0 \\
4 & 1,2 & 2,3 & 1,6 \\
5 & 0,7 & 1,3 & 2,9 \\
Média & 0,7 & 1,5 & 2,0 \\
\hline
\end{tabular}

Comparando-se o grupo em que se usaram suturas (independente do material empregado) com o grupo bipolar verificou-se uma diferença significativa entre as médias dos escores de aderência do ovário direito (Tabela 3 ).
Tabela 2 - Média dos escores de aderências do ovário direito de acordo com o tipo de fechamento do córtex.

\begin{tabular}{|c|c|c|c|}
\hline Tipo de fechamento & Média & $\mathrm{DP}$ & EPM \\
\hline 1. Bipolar $(n=5)$ & 0,7 & 0,2 & 0,1 \\
\hline 2. Poliglactina $(n=5)$ & 1,5 & 0,6 & 0,2 \\
\hline 3. Simples $(n=5)$ & 2,0 & 0,7 & 1,1 \\
\hline \multicolumn{4}{|c|}{$\begin{array}{l}\mathrm{DP}=\text { desvio-padrão do grupo } \\
\text { EPM }=\text { erro-padrão da média } \\
\mathrm{p}=0.02^{*} \text { pela análise de variância (ANOVA) } \\
\text { Teste de Scheffé para comparações múltiplas: }\end{array}$} \\
\hline $\begin{array}{cc}\text { Grupo } 1 \times 2 & \text { Grupo } 1 \times 3 \\
p=0,18 & p=0,02^{*} \\
*=\text { significante } & \end{array}$ & $\begin{array}{c}\text { Grupo } 2 \times 3 \\
p=0,39\end{array}$ & & \\
\hline
\end{tabular}

Tabela 3 - Média dos escores de aderências do ovário direito de acordo com o tipo de fechamento do córtex.

\begin{tabular}{lccc}
\hline Tipo de fechamento & Média & DP & EPM \\
\hline 1. Suturas $(n=10)$ & 1,8 & 0,7 & 0,2 \\
2. Bipolar $(n=5)$ & 0,7 & 0,2 & 0,1 \\
\hline
\end{tabular}

$\mathrm{DP}=$ desvio-padrão do grupo

EPM = erro-padrão da média

$p=0.01^{*}$ pelo teste de $t$ de Student

* $=$ significante

\section{Discussão}

Embora a exérese de cisto ovariano seja uma das cirurgias ginecológicas realizadas com maior freqüência em mulheres jovens, não existe ainda uma técnica padronizada para a reconstituição do ovário. Essas técnicas podem variar no seu potencial de formação de aderências no pós-operatório, muitas vezes causando infertilidade de origem mecânica.

No presente estudo houve a preocupação de estimular o ovário com gonadotrofina coriônica. Este hormônio, além de aumentar o tamanho da gônada, age estimulando os folículos ovarianos e a vascularização do estroma, o que propicia um bom modelo experimental para evidenciar a reação das gônadas não-quiescentes (com cistos) a diversos tipos de fechamento do seu córtex.

No grupo em que não se usou sutura, a hemostasia foi obtida com diatermia bipolar. Esta produz menor área de necrose que a diatermia monopolar, diminuindo a possibilidade do aparecimento de aderências. Nos grupos em que se usou sutura, os fios utilizados foram a poliglactina 910 5-0 e o categute simples 5-0. Esses fios são mais grossos que os fios normalmente usados na microcirurgia (8-0). O objetivo foi de reproduzir ao máximo as condições encontradas na cirurgia ginecológica, na qual o ovário normalmente não é fechado por técnica microcirúrgica em uma 
laparotomia para exérese de cistos de ovário. Nesses dois grupos, não se utilizou qualquer forma de diatermia para obtenção da hemostasia.

Não foram empregadas na cavidade peritoneal substâncias com possivel ação contra a formação de aderências, com o intuito de não introduzir mais uma variável, o que dificultaria a interpretação dos resultados.

Os resultados obtidos no presente estudo estão de acordo com os dados da literatura, ou seja, o fechamento por segunda intenção do córtex ovariano foi mais vantajoso, em relação à formação de aderências, do que o fechamento com o uso de suturas (significativo em relação ao categute simples e com certa tendência em relação à poliglactina 910 5-0). A ausência de significância estatística do grau de aderências entre o grupo em que se usou poliglactina comparado ao grupo controle (fechamento por segunda intenção) pode ter ocorrido pelo baixo poder do estudo, capaz de detectar apenas diferenças de grande magnitude. Ao se comparar o grupo que usou suturas (desconsiderando-se o tipo de fio empregado) com o grupo controle observa-se uma diferença significativa.

Em estudo experimental, Meyer et al. ${ }^{13}$ realizaram uma incisão em ambos os ovários. A hemostasia, invariavelmente de pequena monta, era obtida através da compressão manual. Os ovários dos coelhos foram escolhidos de modo aleatório para serem fechados por primeira intenção com poliglactina 6-0 (sutura contínua, superficial e não entrelaçada) ou deixados para fechar por segunda intenção. Na laparotomia de controle foi verificado menor grau de aderência no grupo em que a lesão fechou por segunda intenção. O fechamento com sutura propiciou um aumento na vascularização das aderências.

Quando se compara o grau de aderência entre os casos com fechamento ovariano por primeira e por segunda intenção em estudos experimentais, verifica-se que o fechamento sem a utilização de suturas causa menos aderências ${ }^{14,15}$. Alguns fios, além de causarem isquemia focal, podem propiciar aderências apenas liberando substâncias irritantes na sua passagem pelo tecido. Hurd et al. ${ }^{16}$, em estudo experimental, demonstraram que o grau de aderência periovariana era maior no lado em que foi utilizada a poliglactina quando comparado com o lado fechado por segunda intenção. Verificaram ainda que no subgrupo no qual foi utilizada a poliglactina, porém com retirada imediata da mesma, o grau de aderência foi similar ao subgrupo que permaneceu com a sutura, sugerindo a liberação de compostos que propiciam a formação de aderências na passagem do fio.
Um dos aspectos importantes é saber se as aderências foram causadas pela cirurgia no ovário ou apenas pela realização da laparotomia. Em todos animais, o ovário esquerdo foi manipulado (não seccionado) pelo mesmo tempo gasto para a realização da cirurgia do ovário oposto. Em apenas dois coelhos foram encontradas aderências frouxas, o que permite afirmar que a laparotomia, por si só, não foi a responsável pela diferença no grau das aderências no ovário operado.

Atualmente, grande parte das cirurgias ovarianas vêm sendo realizadas por via laparoscópica. Sabe-se que a aplicação de suturas na vídeolaparoscopia não é uma tarefa fácil, mesmo para os mais experientes. Com o crescente número de trabalhos mostrando o maleficio das suturas no fechamento do córtex ovariano, a cirurgia laparoscópica para cistos de ovário torna-se menos laboriosa. Em estudo randomizado de laparoscopia versus laparotomia em 28 coelhos, Marana et al. ${ }^{17}$ verificaram que a formação de aderências no pós-operatório, após incisão longitudinal do córtex ovariano (sem uso de suturas), não depende da via de acesso à cavidade peritoneal.

Seria interessante, em novas pesquisas, o estudo do grau de aderência após fechamento do córtex ovariano por segunda intenção (sem uso de suturas), variando-se apenas o tipo de substância intraperitoneal empregada. Celulose oxidada regenerada e o Gore-tex ${ }^{\circledR}$ são agentes de barreira que foram extensamente pesquisados e o seu uso ainda mostra alguns resultados conflitantes. A barreira de hidrogel reabsorvivel foi recentemente testada e parece estar associada a resultados promissores ${ }^{18}$. Além das barreiras, outra substância que pode ser estudada no fechamento do córtex ovariano é a cola biológica (Tissucol®). Ela é basicamente formada de fibrina (soro de vários doadores) e quando misturada à trombina exerce papel hemostático e adesivo ${ }^{19}$.

Embora os dados obtidos em estudos animais não possam ser diretamente transferidos para seres humanos, o modelo animal permite a distribuição aleatória entre os grupos e a formação de um grupo controle apropriado, o que nem sempre é possível conseguir numa pesquisa clínica, onde muitos óbices estão presentes.

\section{SUMMARY}

Purpose: to compare ovarian adhesion according to different types of ovarian cortex closure.

Methods: fifteen rabbits were randomly assigned to 3 groups according to the type of ovarian cortex closure, consisting of 
bipolar diathermy, 5-0 polyglactin 910 and 5-0 plain catgut. After 2 weeks the animals were sacrificed and the adhesion scores were evaluated in the right ovary using the Diamond classification. The left ovary, not incised, was used as control. Statistical analyses were done using analysis of variance (ANOVA), Scheffé test and Student's t test.

Results: the mean adhesion scores for the bipolar, polyglactin and plain catgut groups were 0.7, 1.5 and 2.0, respectively. The analysis of variance detected a significant difference $(p=0.02)$ between the three groups. Using Scheffe test for the two by two comparison of the groups, a significant difference was found between the bipolar and the plain catgut groups. Comparing the group in which sutures were used (independent of the material employed) with the bipolar group, a statistically significant difference was observed (1.8 and 0.7 , respectively, $p=0.01$ ).

Conclusions: the results obtained in this study are in accordance with the literature data, showing that second intention closure is more advantageous, concerning adhesion formation, than closure using sutures.

KEY WORDS: Ovary: adhesions. Sterility.

\section{Referências}

1. Diamond MP, Daniell JF, Martin DC, Feste J, Vaughn WK, McLaughlin DS. Tubal patency and pelvic adhesions at early second-look laparoscopy following intraabdominal use of the carbon dioxide laser: initial report of the intraabdominal laser study group. Fertil Steril 1984; 42:717-23.

2. Diamond MP, Daniell JF, Feste J, et al. Adhesion reformation and de novo formation after reproductive pelvic surgery. Fertil Steril 1987; 47:864-6.

3. Diamond E. Lysis of postoperative pelvic adhesions in infertility. Fertil Steril 1979; 31:287-95.

4. Levinson CJ, Swolin K. Postoperative adhesions: etiology, prevention and therapy. Clin Obstet Gynecol 1980; 23:1213-20.

5. Young PE, Egan JE, Barlow JJ, Mulligan WJ. Reconstructive surgery for infertility at the Boston Hospital for Women. Am J Obstet Gynecol 1970; 108:1092-7.

6. Jessen H. Forty-five operations for sterility. Acta Obstet Gynecol Scand 1971; 50:105-15.

7. Caspi E, Halperin Y, Bukovsky I. The importance of periadnexal adhesion in tubal reconstructive surgery for infertility. Fertil Steril 1979; 31:296300 .
8. Mahadevan MM, Wiseman D, Leader A, Taylor PJ. The effects of ovarian adhesive disease upon follicular development in cycles of controlled stimulation for in vitro fertilization. Fertil Steril 1985; 44:489-92.

9. Diamond MP, Pellicer A, Boyers SP, DeCherney AH. The effect of periovarian adhesions on follicular development in patients undergoing ovarian stimulation for in vitro fertilization-embryo transfer. Fertil Steril 1988; 49:100-3.

10.Hershlag A, Diamond MP, DeCherney AH. Adhesiolysis. Clin Obstet Gynecol 1991; 34:395402.

11.Bronson RA, Wallach EE. Lysis of periadnexal adhesions for correction of infertility. Fertil Steril 1977; 28:613-9.

12.Rapkin AJ. Adhesions and pelvic pain: a retrospective study. Obstet Gynecol 1986; 68:135 .

13. Meyer WR, Grainger DA, DeCherney AH, Lachs MS, Diamond MP. Ovarian surgery on the rabbit: effect of cortex closure on adhesion formation and ovarian function. J Reprod Med 1991; 36:63943.

14.Brumsted JR, Deaton J, Lavigne E, Riddick DH. Postoperative adhesion formation after ovarian wedge resection with and without ovarian reconstruction in the rabbit. Fertil Steril 1990; 53:723-6.

15.Wiskind AK, Toledo AA, Dudley AG, Zusmanis K. Adhesion formation after ovarian wound repair in New Zealand white rabbits: a comparison of ovarian microsurgical closure with ovarian nonclosure. Am J Obstet Gynecol 1990; 163:1674-8.

16.Hurd WW, Himebaugh KS, Cofer KF, Gauvin J, Elkins TE. Etiology of closure-related adhesion formation after wedge resection of the rabbit ovary. J Reprod Med 1993; 38:465-8.

17. Marana R, Luciano AA, Muzii L, Marendino VE, Mancuso S. Laparoscopy versus laparotomy for ovarian conservative surgery: A randomized trial in the rabbit model. Am J Obstet Gynecol 1994; 171:861-4.

18.Hill-West JL, Chowdhury SM, Dunn RC, Hubbell JA. Efficacy of a resorbable hydrogel barrier, oxidized regenerated cellulose, and hyaluronic acid in the prevention of ovarian adhesions in a rabbit model. Fertil Steril 1994; 62:630-4.

19.Larsson B, Jonasson A, Fianu S. Retropubic urethrocystopexy with fibrin sealant: a long-term follow-up. Gynecol Obstet Invest 1988; 26:25761. 